\title{
Nevertrace Claims for Model Checking
}

\author{
Zhe Chen and Gilles Motet \\ LAAS-CNRS, INSA, Université de Toulouse \\ 135 Avenue de Rangueil, 31077 Toulouse, France \\ \{zchen, gilles.motet\}@insa-toulouse.fr
}

\begin{abstract}
In this paper, we propose the nevertrace claim, which is a new construct for specifying the correctness properties that either finite or infinite execution traces (i.e., sequences of transitions) that should never occur. In semantics, it is neither similar to never claim and trace assertion, nor a simple combination of them. Furthermore, the theoretical foundation for checking nevertrace claims, namely the AsynchronousComposition Büchi Automaton Control System (AC-BAC System), is proposed. The major contributions of the nevertrace claim include: a powerful construct for formalizing properties related to transitions and their labels, and a way for reducing the state space in design stage.
\end{abstract}

\section{Introduction}

The SPIN (Simple Promela INterpreter) model checker is an automated tool for verifying the correctness of asynchronous distributed software models [12]3]. System models and correctness properties to be verified are both described in Promela (Process Meta Language). This paper is based on SPIN Version 5.2.5, released on 17th April 2010.

Promela supports various constructs for formalizing different classes of properties. The most powerful constructs are the never claim, the trace and notrace assertion. The never claim specifies the properties on sequences of states, while the trace and notrace assertion specifies the properties on sequences of transitions of simple channel operations, i.e., simple send and receive operations on message channels, where a transition is a statement between two states. However, we observed that the existing constructs cannot specify the properties on full sequences of transitions, apart from the transitions of simple channel operations.

In this paper, we propose the nevertrace claim, which is a new claim construct for specifying correctness properties related to all types of transitions and their labels. A nevertrace claim specifies the properties that either finite or infinite execution traces (i.e., sequences of transitions) that should never occur. A nevertrace claim could be nondeterministic, and performed at every single execution step of the system.

Literally, it seems that the nevertrace claim combines the never claim and the trace assertion. However, we will show that, in semantics, it is neither similar to any of them, nor a simple combination of them. 
The major contributions of this construct include:

First, the nevertrace claim provides a powerful construct for formalizing properties related to transitions and their labels. Furthermore, the nevertrace claim can be used to express the semantics of some existing constructs in Promela.

Second, the nevertrace claim provides a way for reducing the state space in design stage. We observed that variables are always used for two objectives: functional computation, or implicitly recording the execution trace for verification. The nevertrace claim can reduce the usage of variables for marking the execution trace. The decreased number of variables can reduce the state space.

The paper is organized as follows. In Section 2, the existing constructs in Promela are recalled to facilitate further discussion and comparison. In Section 3 , the nevertrace claim is proposed and illustrated by example. The theoretical foundation for checking nevertrace claims, namely the AsynchronousComposition Büchi Automaton Control System (AC-BAC System), is presented in Section 4. Then in Section 5 we show how to express some constructs in Promela using nevertrace claims. We discuss related work in Section 6 and conclude in Section 7 .

To illustrate some constructs in Promela and our new construct in the sequel, we use a simple Promela model (see Listing 1.1) as an example. This model contains two channels (c2s and s2c), three processes (two clients and a server) and four types of messages. Client 1 can send msg1 through the channel c2s, and receive ack1 from the channel $\mathrm{s} 2 \mathrm{c}$, and repeat the procedure infinitely. Client 2 does the similar. There are nine labels in the model, e.g., again at Line 7 , c2srmsg at Line 23. The variable $\mathrm{x}$ counts the number of messages in the channel $c 2 s$, thus is used for functional computation.

Listing 1.1. A Promela Model of Client and Server

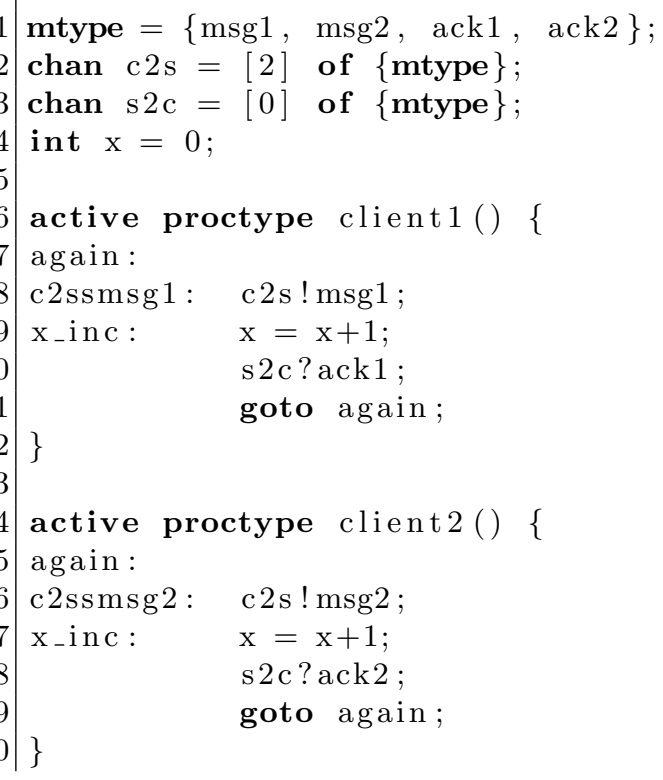




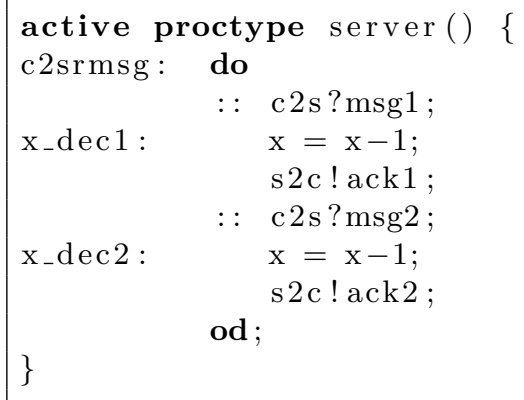

\section{Constructs for Formalizing Properties in SPIN}

Promela supports the following constructs for formalizing correctness properties, of which numerous examples could be found in the monographs 314.

- Basic assertions. A basic assertion is of the form assert(expression).

- End-state labels. Every label name that starts with the prefix end is an end-state label.

- Progress-state labels. Every label name that starts with the prefix progress is a progress-state label.

- Accept-state labels. Every label name that starts with the prefix accept is an accept-state label.

- Never claims. A never claim specifies either finite or infinite system behavior that should never occur.

- Trace assertions. A trace assertion specifies properties about sequences of simple send and receive operations on message channels.

- Notrace assertions. A notrace assertion specifies the opposite of a trace assertion, but uses the same syntax.

Note that never claims could be nondeterministic, whereas trace and notrace assertions must be deterministic. Furthermore, Promela also supports Linear Temporal Logic (LTL) formulas, which are converted into never claims for verification [5].

To facilitate the comparison in the sequel, we recall first the semantics of notrace assertions through a simple example.

The following notrace assertion specifies the property that there should not exist a sequence of send operations on the channel c2s that contains two consecutive c2s!msg1. Note that, for notrace assertions, an error is reported, if the assertion is matched completely. Note that only the send operations on the channel c2s are within the scope of this check, and other statements are ignored.

notrace $\{/ *$ containing two consecutive c2s!msg1 */

S0:

if 


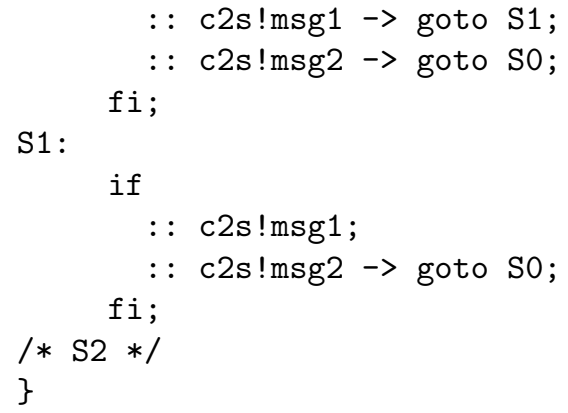

The model in Listing 1.1 violates this assertion, since sequences containing two consecutive c $2 \mathrm{~s}$ ! msg1 are feasible. These sequences cause the termination of the notrace assertion.

We observed that notrace assertions could be also constructed from LTL formulas. The procedure reuses the technique that translates LTL formulas into never claims [5]. Note that never claims specify Nondeterministic Finite Automata (NFA). Thus, an additional step of manually determinizing NFA [6] is needed, since a notrace assertion must be deterministic.

For this example, we can convert the LTL formula $<>(c 2 s$ !msg1 $\rightarrow$ X c2s!msg1) into an NFA in Fig. 1. Note that the condition statement (1) (or true) was replaced by all send operations on the channel c2s in the model. To obtain a deterministic notrace assertion, we have to manually determinize the NFA to a Deterministic Finite Automaton (DFA) and minimize the DFA. The result is shown in Fig. 2, Finally, we can write the above assertion according to the established DFA.

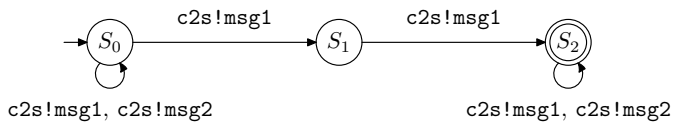

Fig. 1. The Nondeterministic Automaton of notrace Assertion

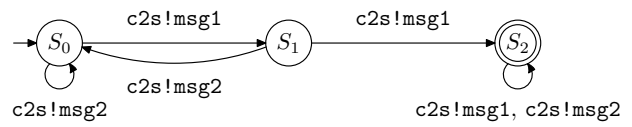

Fig. 2. The Deterministic Automaton of notrace Assertion 


\section{$3 \quad$ Nevertrace Claims}

In this section, we will propose the nevertrace claim. A nevertrace claim may contain only control-flow constructs and transition expressions, while the major ingredient of transition expression is the label expression. Thus, we present three new constructs.

\subsection{Label Expressions}

In a program or model (e.g., Promela model), some statements have labels. A statement may have several labels. A label name in a model contains only the following characters: digits ( 0 to 9 ), letters (a to z, A to Z), and underscore (_). We assume that all the unlabeled statements have the empty string (denoted by $\epsilon)$ as its default label name.

A label expression is a regular expression for matching label names. The label expression reuses a subset of the characters of POSIX-extended regular expressions ${ }^{1}$, and adds some new symbols. The special characters are listed in Table 1. where the additional symbols in the bottom part are not included in POSIX-extended regular expressions.

Note that all characters other than the special characters listed in Table 1. including digits, letters and underscore, match themselves. Also note that the interpretation of these symbols is over the restricted alphabet [0-9A-Za-z_].

\begin{tabular}{|c|c|}
\hline Symbol & Meaning over the Alphabet [0-9A-Za-z_] \\
\hline & A dot matches any single character. \\
\hline$($ ) & Parentheses group a series of patterns into a new pattern. \\
\hline [ ] & $\begin{array}{l}\text { A character class matches any character within the brackets. If the first } \\
\text { character is a circumflex }\left[{ }^{\wedge}\right] \text {, it matches any character except the ones } \\
\text { within the brackets. A dash inside the brackets indicates a character range, } \\
\text { e.g., }\left[\text { a-d] means }[\text { abcd }],\left[{ }^{\wedge} \text { a-d] means }\left[0-9 A-Z e-z_{-}\right] \text {. }\right.\right.\end{array}$ \\
\hline\{\} & $\begin{array}{l}\text { If the braces contain one number, indicate the exact number of times the } \\
\text { previous pattern can match. While two numbers indicate the minimum and } \\
\text { maximum number of times. }\end{array}$ \\
\hline$*$ & A Kleene star matches zero or more copies of the previous pattern. \\
\hline+ & A positive closure matches one or more copies of the previous pattern. \\
\hline$?$ & A question mark matches zero or one copy of the previous pattern. \\
\hline 1 & $\begin{array}{l}\text { An alternation operator matches either the previous pattern or the follow- } \\
\text { ing pattern. }\end{array}$ \\
\hline & Additional Symbols \\
\hline$(\wedge)$ & $\begin{array}{l}\text { If the first character is a circumflex }\left({ }^{\wedge}\right) \text {, it matches any string except the } \\
\text { ones expressed by the expression within the parentheses. }\end{array}$ \\
\hline \# & A hash mark matches any string over the alphabet, i.e., [0-9A-Za-z_] ${ }^{*}$. \\
\hline
\end{tabular}

Table 1. Special Characters in Label Expressions

\footnotetext{
${ }^{1}$ POSIX-extended regular expressions are widely used in Unix applications.
} 
For example, in Listing 1.1, the label expression c2ssmsg\# matches the labels starting with c2ssmsg, i.e., c2ssmsg1 and c2ssmsg2. The expression ( ^ c2ss\#) matches all labels in the model other than c2ssmsg1 and c2ssmsg2. The empty string $\epsilon$ could be matched by a $\{0\}$, where a could be other letters or any digit.

Let us consider the complexity of deciding whether a label name is matched by a label expression. It is well known that a Deterministic Finite Automaton (DFA) can be effectively constructed from a regular expression [6]. In the label expression, most of the special characters are reused from regular expressions. It is easy to see, the additional characters also produce DFA's. For example, ( ${ }^{\wedge}$ ) means constructing the complementation of a regular language (and its DFA) 6. Therefore, a DFA can be also effectively constructed from a label expression.

It is also well known that the membership problem for regular languages (accepted by DFA's) can be decided in linear time [6]. Therefore, the membership problem for label expressions can be also decided in linear time. This means, given a label name $l$ of length $n$, whether $l$ is matched by a label expression can be decided in linear time $O(n)$. This shows that label expressions are feasible in practice.

\subsection{Transition Expressions}

An atomic transition expression is of the form procname [pid] \$1blexp, and may take three arguments. The first optional argument is the name of a previously declared proctype procname. The second optional argument is an expression enclosed in brackets, which provides the process identity number pid of an active process. The third required argument lblexp is a label expression, which matches a set of label names in the model. There must be a symbol $\$$ between the second and the third arguments.

Given a transition and its labels, an atomic transition expression procname [pid] \$1blexp matches the transition (i.e., returns true), if the transition belongs to the process procname[pid], and at least one of the labels is matched by the label expression 1blexp. We should notice that the first two arguments are only used to restrict the application domain of the label expression.

A transition expression contains one or more atomic transition expressions connected by propositional logic connectives. It can be defined in Backus-Naur Form as follows:

$$
t::=a|(! t)|(t \& \& t)|(t|| t)|(t \rightarrow t)
$$

where $\mathrm{t}$ is transition expression, and $\mathrm{a}$ is atomic transition expression.

Given a transition and its labels, a transition expression matches the transition (i.e., returns true), if the propositional logic formula is evaluated to true according to the values of its atomic transition expressions. Note that the transition expression is side effect free. That is, it does not generate new system behavior, just like condition statements.

For example, in Listing 1.1. the (atomic) transition expression client1 [0] \$c2ssmsg\# matches all transitions that have a label starting with c2ssmsg in 
the process 0 of type client1, i.e., the statement with label c2ssmsg1 at Line 8 . The transition expression (client2[1] \$(c2s\#)) \&\& \$again\# matches all transitions that have a label starting with $\mathrm{c} 2 \mathrm{~s}$ and a label starting with again, in the process 1 of type client2, i.e., the statement with two labels again and c2ssmsg2 at Line 16 .

In an atomic transition expression, the second arguments (together with the brackets) can be omitted, if there is only one active process of the type specified by the first argument, or the transition expression is imposed on all active processes of the type. The first and the second arguments (together with the brackets) can be both omitted, if the transition expression is imposed on all active processes. But note that the symbol $\$$ cannot be omitted in any case.

For example, in Listing 1.1, the transition expression client1 [0] \$c2ssmsg\# is equivalent to client $1 \$ c 2$ ssmsg\#. The transition expression $\$ c 2 s s m s g \#$ matches the transitions that have a label starting with c2ssmsg in all active processes, i.e., the statements at Lines 8 and 16 .

The reader may find that the atomic transition expression is syntactically similar to the remote label reference in Promela, except the symbol \$. Note that, at first, there are two superficial differences: (1) the first argument of the remote label reference cannot be omitted, (2) the third argument of the remote label reference should be an existing label name, rather than a label expression. Furthermore, we will show later that they have different semantics in their corresponding claims.

Let us consider the complexity of deciding whether a transition is matched by a transition expression.

For atomic transition expressions, we showed that the membership problem for label expressions (the third argument) can be decided in linear time $O(n)$. As mentioned, the first two arguments only check the owner of the label, so do not affect the complexity. Thus, given a transition with several labels of the total length $n$, whether it is matched by an atomic transition expression can be decided in linear time $O(n)$. That is, the membership problem for atomic transition expressions can be also decided in linear time $O(n)$.

Suppose a transition expression has $i$ atomic transition expressions and $j$ logic connectives, then the membership problem can be decided in $i \cdot O(n)+O(j)$ time. Since $i, j$ are constants for a given transition expression, the membership problem can be decided in linear time $O(n)$. This shows that transition expressions are feasible in practice.

\subsection{Nevertrace Claims}

A nevertrace claim specifies the properties that either finite or infinite execution traces (i.e., sequences of transitions) that should never occur. A nevertrace claim could be nondeterministic, and performed at every single execution step of the system.

A nevertrace claim may contain only control-flow constructs and transition expressions. A nevertrace claim can contain end-state, progress-state and accept-state labels with the usual interpretation in never claims. Therefore, it 
looks like a never claim, except the keyword nevertrace and allowing transition expressions instead of condition statements.

An example of nevertrace claim for the model in Listing 1.1 is as follows:

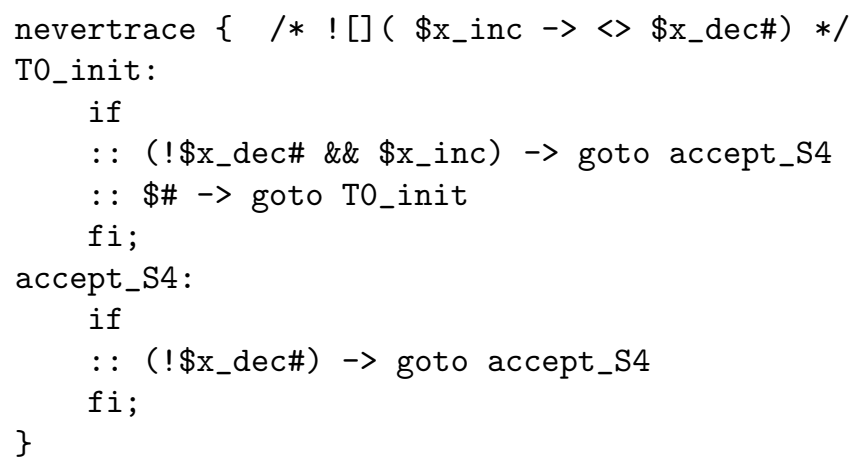

In the example, the claim specifies the property that increasing $\mathrm{x}$ always leads to decrease $\mathrm{x}$ later. In other words, if one of the transitions labeled $\mathrm{x}_{-}$inc is executed, then one of the transitions that have a label starting with $\mathrm{x}_{-}$dec will be executed in the future. By the way, if we replace $\$ \mathrm{x}_{-}$inc by $\$ \mathrm{x}_{-} i \# \mathrm{nc \# \text {, }}$ or replace $\$ \mathrm{x}_{-}$dec\# by server [2] $\$ \mathrm{x}_{-}$dec\#, for this model, the resulting claim is equivalent to the above one.

A nevertrace claim is performed as follows, starting from the initial system state. One transition expression of the claim process is executed each time after the system executed a transition. If the transition expression matches the last executed transition, then it is evaluated to true, and the claim moves to one of the next possible statements. If the claim gets stuck, then this means that the undesirable behavior cannot be matched. Therefore, no error is reported.

For a rendezvous communication, the system executes an atomic event in which two primitive transitions are actually executed at a time, one send operation and one receive operation. In this case, we assume that the send operation is executed before the receive operation in an atomic rendezvous event.

An error is reported, if the full behavior specified could be matched by any feasible execution. The violation can be caught as termination of the claim, or an acceptance cycle, just like never claims. Note that all the transitions of the model are within the scope of the check.

In the example, it is easy to see that there exists no violation, since the nevertrace claim cannot be matched completely.

Note that it is hard to express this property using existing constructs in Promela. For example, trace or notrace assertions are not capable of expressing this property, since they can only specify properties on simple channel operations. The three types of special labels do not have this power neither.

Fortunately, never claims can express this property by introducing new variables to implicitly record the information about execution traces. For instance, we introduce two boolean variables a, b. After each statement labeled $\mathrm{x}_{-}$inc, we add the statements " $\mathrm{a}=1 ; \mathrm{a}=0$;", which let a be 1 once. After each statement labeled $x_{-}$dec\#, we add the statements " $\mathrm{b}=1 ; \mathrm{b}=0 ;$;", which let $\mathrm{b}$ be 1 
once. Then the property can be expressed as LTL formula [] (a $-><>b)$. The negation of this formula can be converted into a never claim that specifies the required property.

However, please note that the additional variables quadruple the state space (different combinations of $\mathrm{a}$ and $\mathrm{b}$ ), and make the program malformed and harder to read. In contrast, the nevertrace claim is more economic, since it takes full advantage of the transition information (e.g., control-flow states and their labels) that is already tracked as part of the state space in the verification mode of SPIN.

An interesting thing is that nevertrace claims could be also converted from LTL formulas. In the example, a never claim can be generated from the LTL formula ! [] (\$x_inc $\rightarrow<>\$ x_{-}$dec\#) by SPIN. Then we can obtain the nevertrace claim above by replacing the condition statement (1) or true by \$\# matching all transitions. This fact can facilitate the use of nevertrace claims in practice.

Finally, let us consider the syntax definition of nevertrace claims. There are various ways to modify the grammar of Promela to take into account the nevertrace claim. For example, we can add the following productions into the grammar of Promela.

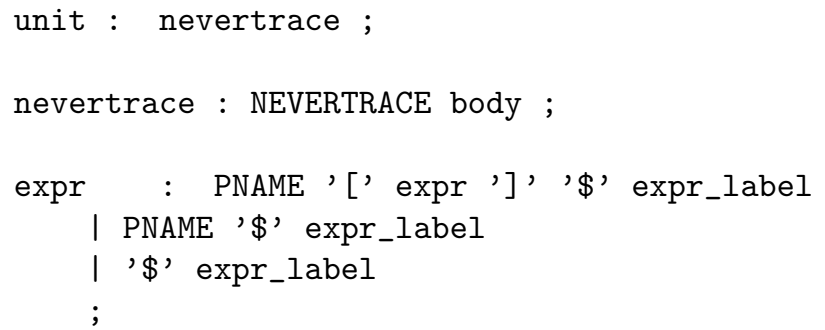

Note that unit, body and expr are existing nonterminals in the grammar of Promela, thus we only append the new productions. The productions for the nonterminal expr_label are omitted, since its syntax is clearly specified in Table 1 .

\section{Theoretical Foundation for Checking Nevertrace Claims}

In this section, we propose the theory of asynchronous-composition Büchi automaton control systems. Then we will show the connection between the theory and the checking of nevertrace claims by example.

\subsection{The Asynchronous Composition of Büchi Automata}

At first, we recall the classic definition of Büchi automata [7/8].

Definition 1. A (nondeterministic) Büchi automaton (simply automaton) is a tuple $A=\left(Q, \Sigma, \delta, q_{0}, F\right)$, where $Q$ is a finite set of states, $\Sigma$ is a finite alphabet, 
$\delta \subseteq Q \times \Sigma \times Q$ is a set of named transitions, $q_{0} \in Q$ is the initial state, $F \subseteq Q$ is a set of accepting states. For convenience, we denote the set of transition names also by $\delta$.

Note that the concept of transition name is introduced. A transition in $\delta$ is of the form $p_{k}:\left(q, a, q^{\prime}\right)$, where $p_{k}$ is the name of the transition. In the transition diagram, a transition $p_{k}:\left(q, a, q^{\prime}\right) \in \delta$ is denoted by an arc from $q$ to $q^{\prime}$ labeled $p_{k}: a$.

Given a set of automata, they execute asynchronously, but may synchronize on rendezvous events. We assume that the send operation is executed before the receive operation in an atomic rendezvous event. Formally, we define their asynchronous composition as follows.

Let $N=\left\{n_{1}, \ldots, n_{k}\right\} \subseteq \mathbb{N}$ be a countable set with cardinality $k$, and for each $n_{j} \in N, S_{n_{j}}$ be a set. We define the Cartesian product as:

$$
\prod_{n_{j} \in N} S_{n_{j}}=\left\{\left(x_{n_{1}}, x_{n_{2}}, \ldots, x_{n_{k}}\right) \mid \forall j \in\{1, \ldots, k\}, x_{n_{j}} \in S_{n_{j}}\right\}
$$

For each $j \in\{1, \ldots, k\}$, we denote the $j$-th component of the vector $q=\left(x_{n_{1}}, x_{n_{2}}\right.$, $\left.\ldots, x_{n_{k}}\right)$ by the projection $q[j]$, i.e., $q[j]=x_{n_{j}}$.

Definition 2. The asynchronous composition $A=\prod_{n \in N} A_{n}$ of a countable collection of Büchi automata $\left\{A_{n}=\left(Q_{n}, \Sigma_{n}, \delta_{n}, q_{n}, F_{n}\right)\right\}_{n \in N}$ is a Büchi automaton

$$
A=\left(\prod_{n \in N} Q_{n}, \Sigma, \delta, \prod_{n \in N} q_{n}, F\right)
$$

where $\Sigma=\bigcup_{n \in N} \Sigma_{n}$,

$$
\begin{gathered}
\delta=\left\{p_{k}:\left(q, a, q^{\prime}\right) \mid \exists n_{i} \in N, a \in \Sigma_{n_{i}} \wedge p_{k}:\left(q[i], a, q^{\prime}[i]\right) \in \delta_{n_{i}},\right. \\
\text { and } \left.\forall n_{j} \in N, n_{j} \neq n_{i} \rightarrow q[j]=q^{\prime}[j]\right\}
\end{gathered}
$$

$F=\left\{q \in \prod_{n \in N} Q_{n} \mid \exists n_{i} \in N, q[i] \in F_{n_{i}}\right\}$.

We interpret the asynchronous composition as the expanded version which fully expands all possible values of the variables in the state space. The executability of a transition depend on its source state.

\subsection{The Asynchronous-Composition BAC System}

An Asynchronous-Composition Büchi Automaton Control System (AC-BAC System) consists of an asynchronous composition of Büchi automata and a Büchi controlling automaton. The controlling automaton controls all the transitions of the primitive components in the composition. Thus, the alphabet of the controlling automaton equals the set of transition names of the controlled automata.

Definition 3. Given an asynchronous composition of a set of Büchi automata $A=\prod_{n \in N} A_{n}, a$ (Büchi) controlling automaton over $A$ is $A_{c}=\left(Q_{c}, \Sigma_{c}, \delta_{c}, q_{c}, F_{c}\right)$ with $\Sigma_{c}=\bigcup_{n \in N} \delta_{n}$. The global system is called an Asynchronous-Composition Büchi Automaton Control System (AC-BAC System). 
The controlling automaton is used to specify the sequences of transitions that should never occur, since the $\omega$-words accepted by a controlling automaton are sequences of transitions.

We compute the meta-composition of an asynchronous composition and a controlling automaton. In the meta-composition, a transition is allowed iff it is in the asynchronous composition and allowed by the controlling automaton. The name "meta-composition" denotes that the controlling automaton is at a higher level, since it treats the set of transitions rather than the alphabet. We formally define the meta-composition $(\vec{\bullet}$ operator) as follows.

Definition 4. The meta-composition of an asynchronous composition $A=(Q$, $\left.\Sigma, \delta, q_{0}, F\right)$ and a controlling automaton $A_{c}=\left(Q_{c}, \Sigma_{c}, \delta_{c}, q_{c}, F_{c}\right)$ is a Büchi automaton:

$$
A^{\prime}=A \vec{P} A_{c}=\left(Q \times Q_{c}, \Sigma, \delta^{\prime},\left(q_{0}, q_{c}\right),\left(F \times Q_{c}\right) \cup\left(Q \times F_{c}\right)\right)
$$

where for each $q_{i}, q_{l} \in Q, q_{j}, q_{m} \in Q_{c}$ and $a \in \Sigma$, we have $p_{k}:\left(\left(q_{i}, q_{j}\right), a,\left(q_{l}, q_{m}\right)\right) \in$ $\delta^{\prime}$ iff $p_{k}:\left(q_{i}, a, q_{l}\right) \in \delta$ and $\left(q_{j}, p_{k}, q_{m}\right) \in \delta_{c}$.

If we let $\left\{A_{n}\right\}_{n \in N}$ contain only a single primitive automaton, i.e., $|N|=1$, the definition will express the meta-composition over a single automaton. This means, the controlling automaton over a single automaton is only a special case of the above definition.

We observe that $A$ has feasible sequences of transitions accepted by $A_{c}$ (or $A$ matches $A_{c}$ ), iff the language accepted by the meta-composition is not empty. This fact will be used for checking nevertrace claims.

Note that the emptiness problem for meta-compositions is decidable. It is well known that the emptiness problem for Büchi automata is decidable [8]. Since the meta-composition is a Büchi automaton, checking its emptiness is also decidable.

\subsection{From Nevertrace Claims to AC-BAC Systems}

We will show at first how to translate a model with a nevertrace claim into an AC-BAC system. Let us consider a simple example in Listing 1.2 consisting of a client and a server.

Listing 1.2. A Simplified Promela Model of Client and Server

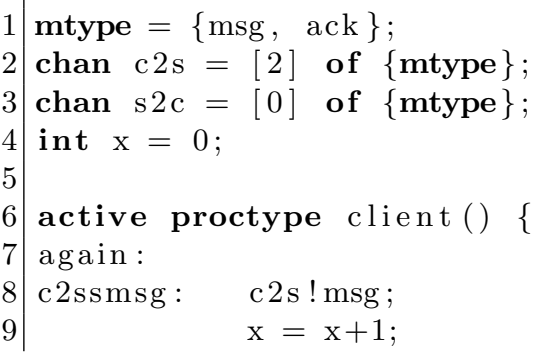




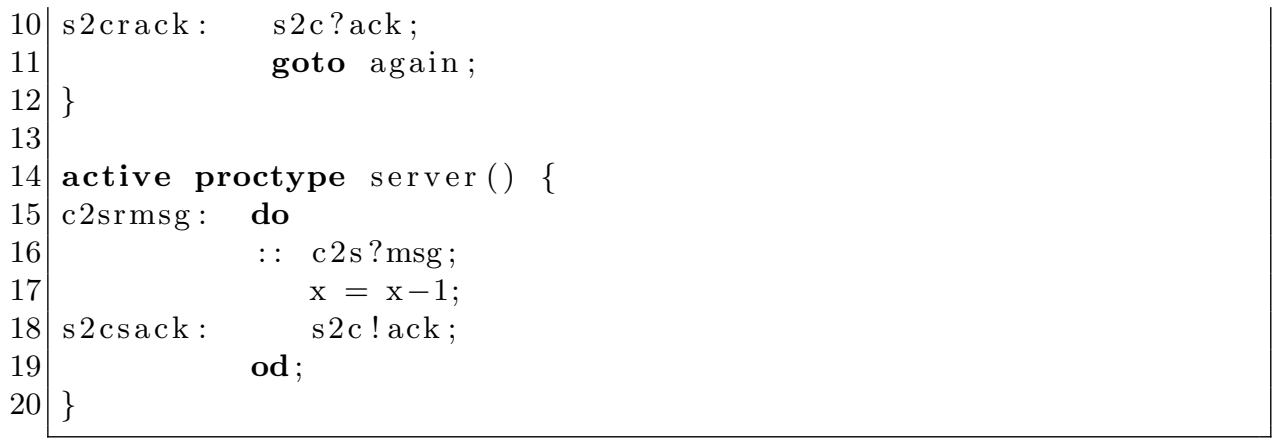

Figures 3 (1) and (2) show the automata $A_{1}, A_{2}$ describing the behavior of the client and the server, respectively. Each transition between two control-flow states is labeled by its transition name (may be related to line number and control-flow state etc.) and statement. For example, 8 is the transition name between the states 8 and 9 .

The asynchronous composition $A_{1} \cdot A_{2}$ is shown in Fig. 3.3). A system state consists of four elements: the control-flow states of $A_{1}$ and $A_{2}$, the value of $\mathrm{x}$ and the contents of the channel $\mathrm{c} 2 \mathrm{~s}$. Note that the dashed transitions are not executable at their corresponding states, although themselves and omitted subsequent states are part of the composition. That is, the figure contains exactly the reachable states in the system. At the bottom of the figure, the transitions labeled 18 and 10 constitute a handshake.

Let us consider the following nevertrace claim:

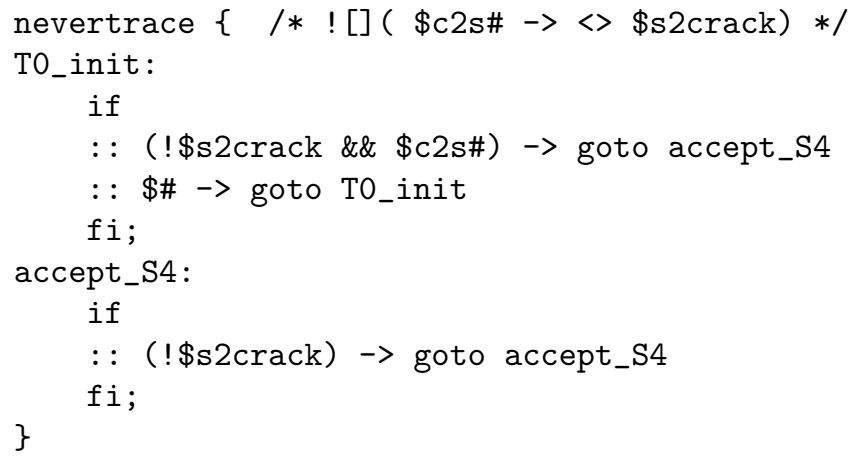

The claim specifies the property that any operation on channel c2s always leads to receive ack from the channel s2c later. In other words, if one of the transitions that have a label starting with $\mathrm{c} 2 \mathrm{~s}$ is executed, then one of the transitions labeled s2crack will be executed in the future.

Figure 3(4) shows the automaton specified by the nevertrace claim. Figure $3(5)$ shows the controlling automaton specified by the nevertrace automaton. For example, the transition expression (! \$s2crack \&\& \$c2s\#) matches the transitions 8 and 16 . 
The automata in Figures 3(3) and (5) constitute an AC-BAC system, which is established from the model and the nevertrace claim. The next step is to check whether the asynchronous composition $A_{1} \cdot A_{2}$ matches the claim $A_{c}$.

The meta-composition $\left(A_{1} \cdot A_{2}\right) \vec{\cdot} A_{c}$ is shown in Fig. 3(6) (the state space starting from the state $\left(9,15, q_{0}\right)$ is not drawn). The state of the controlling automaton is added into the system state. In the meta-composition, a transition is allowed, iff it is in $\left(A_{1} \cdot A_{2}\right)$ and allowed by $A_{c}$. Note that the transition 10 is blocked, since it is not allowed by the state $q_{1}$ of $A_{c}$. It is easy to see there does not exist any acceptance cycle, thus the $\omega$-language accepted by the metacomposition is empty. This means, no counterexample can be found, and the system satisfies the required correctness property.

To conclude, checking nevertrace claims is equivalent to the emptiness problem for meta-compositions. A nevertrace claim is violated if and only if the meta-composition is not empty. As we mentioned, the emptiness problem for meta-compositions is decidable. Therefore, checking nevertrace claims is feasible in practice. Furthermore, the emptiness of meta-compositions can be checked on-the-fly, using the technique for checking never claims.

\section{On Expressing Some Constructs in SPIN}

In this section, we will show how to express the semantics of some constructs in Promela using nevertrace claims, although the nevertrace claim does not aim at replacing them.

\subsection{Expressing Notrace Assertions}

There are various ways to convert a notrace assertion into a nevertrace claim. As an example, let us consider the notrace assertion in Section 2. We can construct an NFA in Fig. 4 which specifies the same property as the DFA in Fig. 2 of the notrace assertion for the model in Listing 1.1 .

A nevertrace claim can be written according to the automaton.

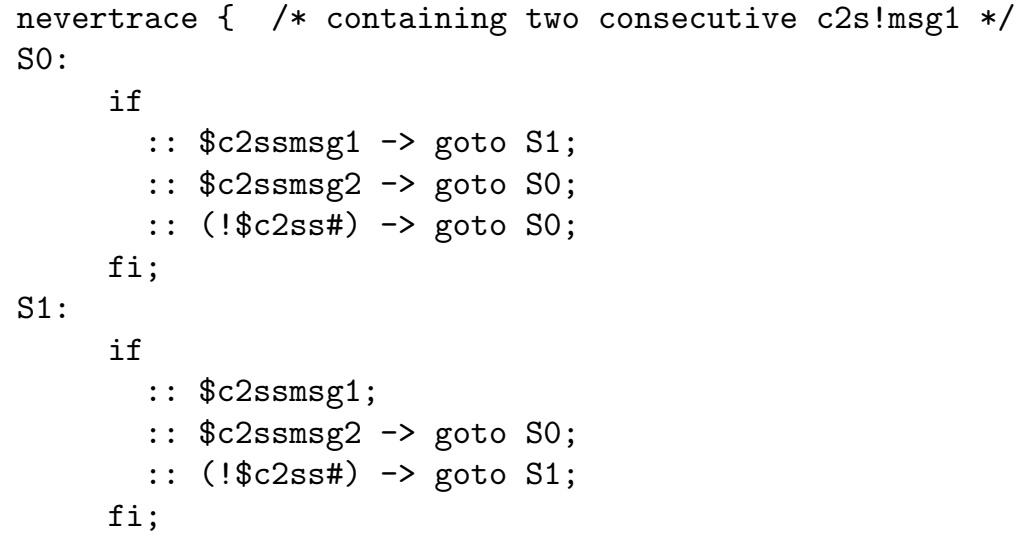


It is easy to see, we used the following rules to convert the notrace assertion into a nevertrace claim.

First, in the system model, all send operations of message msg on a channel ch must have a label chsmsg, while all receive operations of message msg on the channel must have a label chrmsg. The labels of all statements other than channel operations should not start with the names of declared channels.

Second, in the notrace assertion, (1) replace ch!msg by $\$$ chsmsg, ch?msg by $\$$ chrmsg; (2) for each state, add new transition expressions to match the statements outside the scope of the notrace assertion. In the example, for each state, we add a transition from the state to itself with the transition expression ( $\$$ \$c2ss\#), since only send operations on the channel c2s are within the scope of the notrace assertion.

\subsection{Expressing Remote Label Reference}

The predefined function procname[pid]@label is a remote label reference. It returns a nonzero value only if the next statement that can be executed in the process procname [pid] is the statement with label.

It seems that procname[pid]@label can be also used as a transition expression, if replacing @ by $\$$. However, there is a little difference in semantics. The remote label reference is evaluated over the next statement of the process procname [pid], but the transition expression is evaluated over the last executed transition, which does not necessarily belong to the process procname [pid].

\subsection{Expressing the Non-Progress Variable}

The predefined non-progress variable $\mathrm{np}_{-}$holds the value false, if at least one running process is at a control-flow state with a progress-state label. It is used to detect the existence of non-progress cycles.

It seems that the variable $\mathrm{np}_{-}$is equivalent to our transition expression ! \$progress\#. However, there is a little difference. The variable $\mathrm{np}$ - is evaluated over all the running processes, but the transition expression is evaluated over the last executed process.

\subsection{Expressing Progress-State Labels}

There are two types of progress cycles. A weak progress cycle is an infinite execution cycle that contains at least one of the progress-state labels, which denotes reaching some progress-state labels infinitely often. A strong progress cycle is a weak progress cycle with the requirement that each statement with a progress-state label in the cycle must be executed infinitely often.

Promela supports only the weak progress cycle, whereas our nevertrace claim can express the strong progress cycle. 
As an example, let us consider the model in Listing 1.3 consisting of two processes $\mathrm{p} 1$ and $\mathrm{p} 2$. Note that $\mathrm{p} 1$ does not execute any statement, but waits at the label progress for ever.

Listing 1.3. A Promela Model of Two Processes

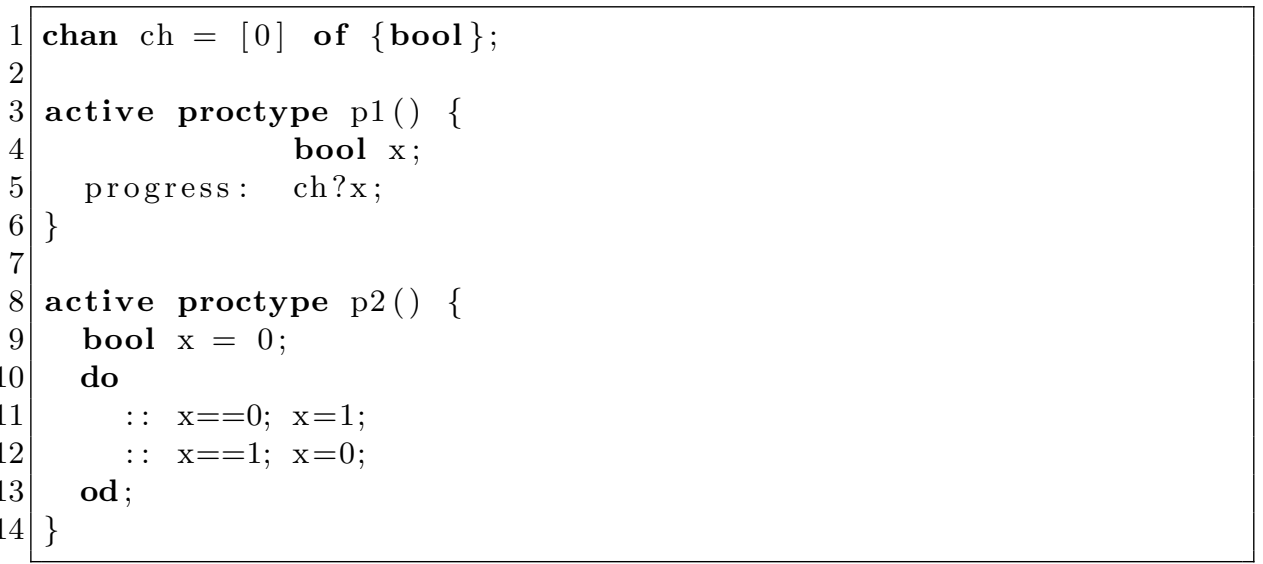

In the verification mode of SPIN, none (weak) non-progress cycle is found, both with or without fairness condition. All executions are weak progress cycles, since 1 stays for ever at a progress-state label.

In contrast, we can find a strong non-progress cycle using the following nevertrace claim, which can be constructed from the LTL formula $<>$ [] ! \$progress\#. If we modify a never claim generated from the LTL formula by SPIN, remember to replace the condition statement (1) or true by $\$$ \# matching all transitions.

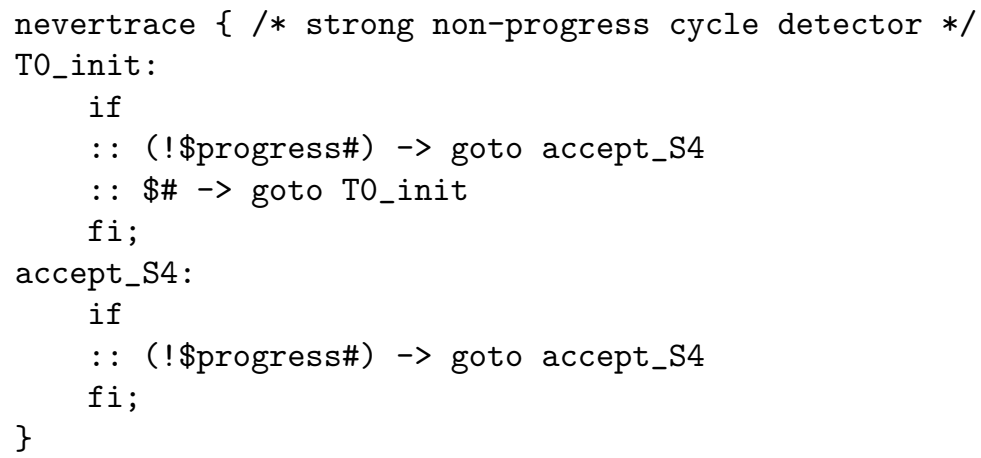

Note that the evaluation of transition expressions is over the last executed transition, and all the executable transitions do not have a progress-state label. Therefore, strong non-progress cycles can be detected as counterexamples.

\subsection{Expressing Accept-State Labels}

There are two types of acceptance cycles. A weak acceptance cycle is an infinite execution cycle that contains at least one of the accept-state labels, which 
denotes reaching some accept-state labels infinitely often. A strong acceptance cycle is a weak acceptance cycle with the requirement that each statement with an accept-state label in the cycle must be executed infinitely often.

Promela supports only the weak acceptance cycle, whereas our nevertrace claim can express the strong acceptance cycle.

As an example, let us replace the label progress by accept in the model of Listing 1.3 . Note that $\mathrm{p} 1$ does not execute any statement, but waits at the label accept for ever.

In the verification mode of SPIN, a (weak) acceptance cycle is found, both with or without fairness condition. All executions are weak acceptance cycles, since $\mathrm{p} 1$ stays for ever at an accept-state label. Therefore, (weak) acceptance cycles can be detected as counterexamples.

In contrast, we cannot find any strong acceptance cycle using the following nevertrace claim, which can be constructed from the LTL formula [] <> (\$accept\#). If we modify a never claim generated from the LTL formula by SPIN, remember to replace the condition statement (1) or true by $\$ \#$ matching all transitions.

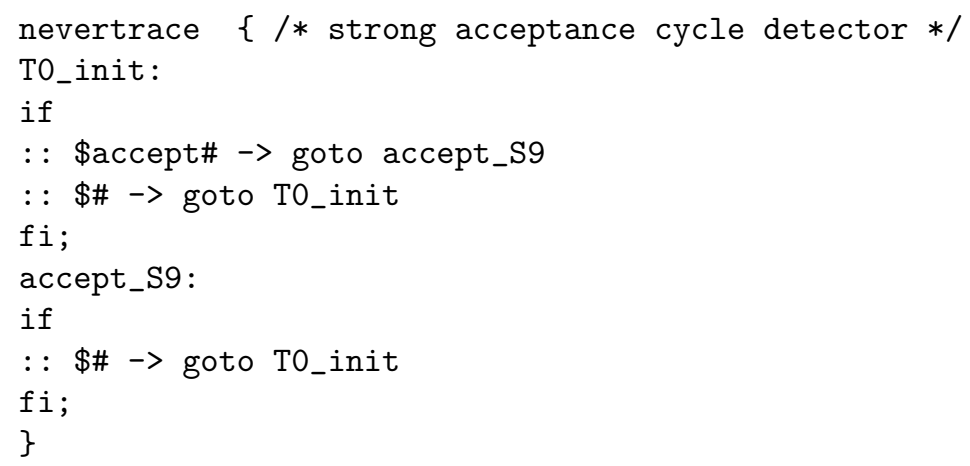

Note that the evaluation of transition expressions is over the last executed transition, and all the executable transitions do not have an acceptance-state label. Therefore, there is no strong acceptance cycle.

\section{Related Work}

In the previous section, we mentioned some differences between nevertrace claims and some constructs in Promela. In this section, we would like to summarize the comparison with the most powerful two constructs in Promela, never claims and notrace assertions.

The major differences between nevertrace claims and never claims are obvious. They are used to specify properties on sequences of transitions (execution traces) and sequences of states, respectively. A nevertrace claim is performed after executing a transition, whereas a never claim is started from the initial system state. A nevertrace claim is evaluated over the last executed transition, whereas a never claim is evaluated over the current system state. Thanks to 
their different focuses, they can be used together in a model checker to achieve stronger power.

Nevertrace claims and notrace (also trace) assertions are both about execution traces, their major differences are as follows.

1. They have different scopes of checking. Nevertrace claims consider all transitions, whereas only simple send/receive operations are within the scope of notrace assertions. Furthermore, only the channel names that are specified in a notrace assertion are considered to be within its scope. All other transitions are ignored.

2. The notrace assertion cannot contain random receive, sorted send, or channel poll operations. But these can be also tracked by a nevertrace claim.

3. The notrace assertion must be deterministic, whereas the nevertrace claim could be nondeterministic, just like the never claim.

4. The notrace assertion does not execute synchronously with the system, but executes only when events of interest occur. Whereas the notrace assertion executes synchronously with the system, just like the never claim.

\section{Conclusion}

In this paper, we proposed the nevertrace claim, which is a new construct for specifying the correctness properties that either finite or infinite execution traces (i.e., sequences of transitions) that should never occur. The AsynchronousComposition Büchi Automaton Control System (AC-BAC System) provides the theoretical foundation for checking nevertrace claims.

\section{References}

1. Holzmann, G.J., Peled, D.: The state of SPIN. In Alur, R., Henzinger, T.A., eds.: Proceedings of the 8th International Conference on Computer Aided Verification (CAV 1996). Volume 1102 of Lecture Notes in Computer Science., Springer (1996) 385-389

2. Holzmann, G.J.: The model checker SPIN. IEEE Transactions on Software Engineering 23(5) (1997) 279-295

3. Holzmann, G.J.: The SPIN Model Checker: Primer and Reference Manual. AddisonWesley Professional (2003)

4. Ben-Ari, M.: Principles of the SPIN Model Checker. Springer (2008)

5. Gastin, P., Oddoux, D.: Fast LTL to Büchi automata translation. In: Proceedings of the 13th International Conference on Computer Aided Verification (CAV'01). Volume 2102 of Lecture Notes in Computer Science., Springer (2001) 53-65

6. Hopcroft, J.E., Ullman, J.D.: Introduction to Automata Theory, Languages, and Computation. Addison-Wesley, Reading MA (1979)

7. Büchi, J.R.: On a decision method in restricted second order arithmetic. In: Proceedings of the International Congress on Logic, Methodology, and Philosophy of Science, Stanford University Press (1960) 1-11

8. Thomas, W.: Automata on infinite objects. In: Handbook of Theoretical Computer Science (vol. B): Formal Models and Semantics, Elsevier Science Publishers B.V. (1990) 133-191 

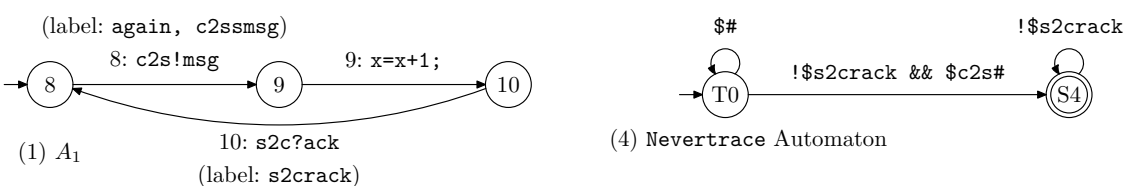

(4) Nevertrace Automaton
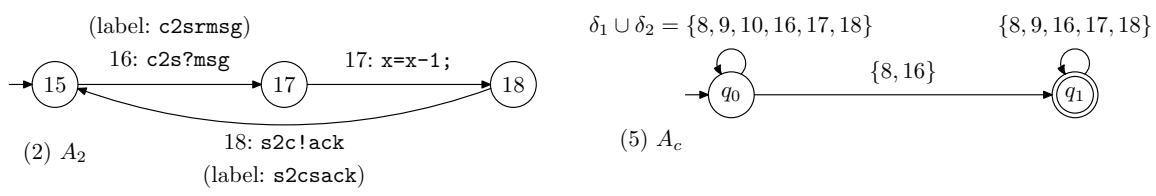

(5) $A_{c}$
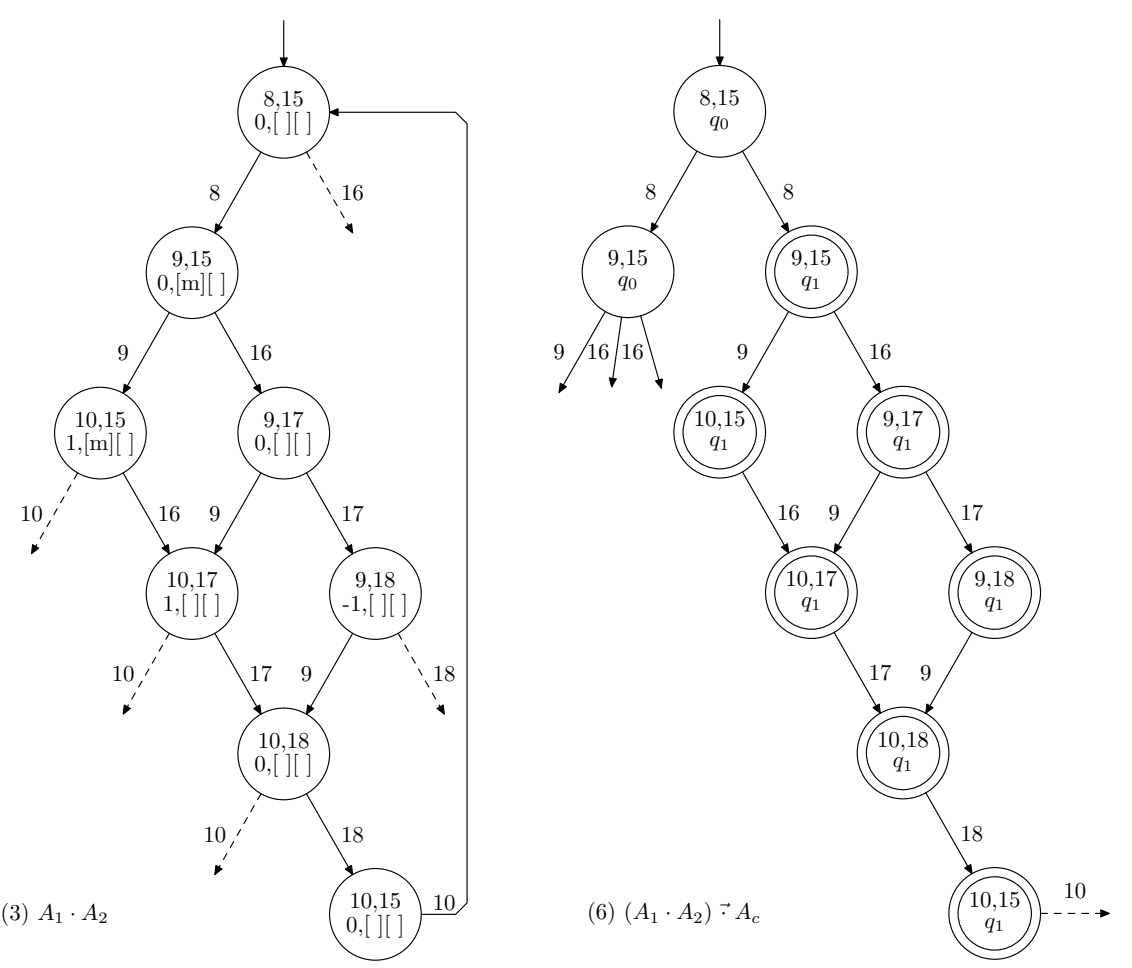

Fig. 3. An Example of Asynchronous-Composition BAC System

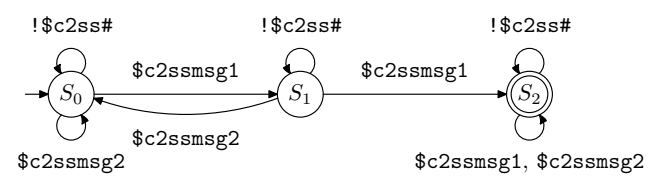

Fig. 4. The Nondeterministic Automaton of nevertrace Claim 